\title{
Vereinbarkeit des neuen Brennstoffemissionshandelsgesetzes mit der Finanzverfassung
}

\author{
Lucas Hennicke*
}

(c) Der/die Autor(en) 2021. Dieser Artikel ist eine Open-Access-Publikation.

\begin{abstract}
Das neue Brennstoffemissionshandelsgesetz (BEHG) ${ }^{1}$ soll durch einen ab 2021 greifenden Preisanreiz dafür sorgen, dass vor allem in den Bereichen Verkehr und Wärme $\mathrm{CO}_{2}$-Emissionen eingespart werden. Zweifel an der Vereinbarkeit mit der Finanzverfassung wurden allerdings laut an der sog. Einführungsphase, die einen Verkauf der Zertifikate zu einem Fixpreis vorsieht, sowie an der anschließenden Übergangsphase, in der eine Versteigerung innerhalb eines Preiskorridors mit Unter- und Obergrenze stattfindet. ${ }^{2}$ Dieser Aufsatz möchte zeigen, dass das BEHG in der bestehenden Abgabensystematik verortet und gerechtfertigt werden kann. Hierfür werden die für die finanzverfassungsrechtliche Bewertung relevanten Regelungsinstrumente des BEHG dargestellt (1), geprüft, ob sich hinter dem Fixpreis $b z w$. der Preisobergrenze eine Steuer verbirgt (2) und schließlich eine Einordnung als nichtsteuerliche Abgabe und deren Rechtfertigung thematisiert (3).
\end{abstract}

\section{Ziele, Struktur und Regelungsinstrumente des BEHG}

Nach $\int 1$ S. 1 BEHG ist es Zweck des Gesetzes, eine „Grundlage für den Handel mit Zertifikaten für Emissionen aus Brennstoffen zu schaffen und für eine Bepreisung dieser Emissionen zu sorgen". Das BEHG sieht folglich die Einführung eines ökonomischen Anreizinstrumentes in Form eines Handelssystems vor, bei dem die absolut zulässige Menge der $\mathrm{CO}_{2}$-Emissionen zumindest im Grundsatz (vgl. \$3 Nr. $1 \mathrm{BEHG}$ ) begrenzt und in handelbare Zertifikate unterteilt wird. ${ }^{3}$ So soll ein marktbasierter Anreiz geschaffen werden, Emissionen aus der Verbrennung der erfassten Stoffe ${ }^{4}$ einzusparen.

\subsection{Ziele des BEHG}

Durch das Handelssystem sollen die nationalen Klimaschutzziele sowie das „langfristige Ziel der Treibhausgasneutralität bis 2050 " (vgl. $\$ 1$ S. 1 BEHG) erreicht werden. Die nationalen Klimaschutzziele ergeben sich aus $\$ 3$ Abs. 1 Bundes-Klimaschutzgesetz (KSG) ${ }^{5}$. Demnach sollen die Treibhausgasemissionen schrittweise im Vergleich zum Jahr 1990 gemindert werden. Nach Satz 2 gilt bis zum Zieljahr 2030 eine Minderungsquote von mindestens 55\%. Ein ausdrückliches Verbot der Abschwächung der Ziele statuiert $\$ 3$ Abs. 3 S. 2 KSG.

Zudem soll das BEHG zum Erreichen der Minderungsziele nach der EU-Klimaschutzverordnung beitragen. ${ }^{6}$ Deutschland trifft (noch) ${ }^{7}$ eine Reduktionsverpflichtung von 38\% ggü. 2005 im Jahr 2030 für Treibhausgasemissionen $^{8}$, die den IPCC-Quellenkategorien Energie, Industrieprozesse und Produktverwendung, Landwirtschaft und Abfall gemäß der Verordnung (EU) Nr. 525/20139 zuzuordnen sind und nicht bereits vom Europäischen Emis-

Lucas Hennicke,

Doktorand im BMBF-geförderten Kompetenznetzwerk

„Zukunftsherausforderungen des Umweltrechts“" (KomUR),

am Institut für Umwelt- und Planungsrecht

bei Prof. Dr. Sabine Schlacke,

Westfälische Wilhelms-Universität Münster,

Münster, Deutschland sionshandel (EU-ETS) erfasst werden ${ }^{10}$. Das BEHG hat keinen eindeutigen sektoralen Bezug, da es an das Inverkehrbringen der Brennstoffe anknüpft. Es kann somit potentiell auch bereits dem EU-ETS unterliegende Anlagen in den Sektoren Energieerzeugung und der energieintensiven Industrie sowie den Luftverkehr ${ }^{11}$ indirekt betreffen, wenn diese durch den Kauf von entsprechenden Brennstoffen dem erhöhten Preissignal ausgesetzt sind. Doppelbelastungen sollen allerdings vermieden ( $\$ 7$ Abs. 5 BEHG)

*) Der Autor dankt Helen Wentzien für die inhaltliche Diskussion und Anmerkungen bei dem Verfassen des Aufsatzes.

1) Gesetz über einen nationalen Zertifikatehandel für Brennstoffemissionen v. 12.12.2019, BGB1. I S. 2728.

2) Siehe z.B.: IKEM/Rodi, Verfassungsmäßigkeit des Entwurfs zum Brennstoffemissionshandelsgesetz (BEHG-E), November 2019, S. $12 \mathrm{ff}$.; Müller/Kahl, Zur verfassungsrechtlichen Einordnung des Brennstoffemissionshandelsgesetzes - Stellungnahme zur Anhörung des Ausschusses für Umwelt, Naturschutz und nukleare Sicherheit im Deutschen Bundestag am 6.11.2019, Würzburger Berichte zum Umweltenergierecht, Nr. 45, 4.11.2019, S. $2 \mathrm{ff}$; Wernsmann/Bering, NVwZ 2020, 497; Klinski/Keimeyer sehen eine Vereinbarkeit mit der Finanzverfassung nur, wenn das BVerfG seine bisherige Argumentationslinie erweitere: ZUR $2020,342,348$.

3) Vgl. Gesetzentwurf der Fraktionen CDU/CSU und SPD, Entwurf eines Gesetzes über einen nationalen Zertifikatehandel für Brennstoffemissionen (Brennstoffemissionshandelssystem - BEHG) v. 5.11.2019, BT-Drs. 19/14746 (im Folgenden: BTDrs. 19/14746), S. 20 f.; siehe zur Funktionsweise auch: Vollmer, NuR 2020, 237.

4) Das BEHG erfasst das Inverkehrbringen aller Kraftstoffe und kohlenwasserstoffbasierter Heizstoffe (Anlage 1 S. $2 \mathrm{zu} \$ 2$ Abs. 1 BEHG) mit Ausnahme von Torf und Waren der Position 4401 und 4402 der Kombinierten Nomenklatur (S. 2) sowie Waren, die vom Alkoholsteuergesetz erfasst werden (S. 3). Für die Jahre 2021-2022 werden allerdings nur Ottokraftstoffe, Diesel, Erdgas und Heizöl (\$7 Abs. 2 S. 2 i. V.m. Anlage 2 BEHG) berücksichtigt, vgl. dazu auch: BT-Drs. 19/14746, S. 35.

5) Gesetz zur Einführung eines Bundes-Klimaschutzgesetzes und zur Änderung weiterer Vorschriften v. 12.12.2019, BGBl. I S. 2513.

6) $\$ 3$ Nr. 5 BEHG definiert die EU-Klimaschutzverordnung als ,die Verordnung (EU) 2018/842 des Europäischen Parlaments und des Rates vom 30.5.2018 zur Festlegung verbindlicher nationaler Jahresziele für die Reduzierung der Treibhausgasemissionen im Zeitraum 2021 bis 2030 als Beitrag zu Klimaschutzmaßnahmen zwecks Erfüllung der Verpflichtungen aus dem Übereinkommen von Paris sowie zur Änderung der Verordnung (EU) Nr. 525/2013 (ABl. L 156 vom 19.6.2018, S. 26) in der jeweils geltenden Fassung".

7) Im Zuge des Green Deal sollen auch die Ziele der EU-Klimaschutzverordnung angepasst werden, vgl. Komm., Mitt. v. 11.12.2019, Der europäische Grüne Deal, Anhang, KOM(2019) 640 endg.

8) Art. 4 Abs. 1 VO i. V.m. Anhang I VO (EU)2018/842

9) Verordnung (EU) Nr. 525/2013 des Europäischen Parlaments und des Rates vom 21.5.2013 über ein System für die Überwachung von Treibhausgasemissionen sowie für die Berichterstattung über diese Emissionen und über andere klimaschutzrelevante Informationen auf Ebene der Mitgliedstaaten und der Union und zur Aufhebung der Entscheidung Nr. 280/2004/EG. 10) Art. 2 Abs. 1 VO (EU)2018/842.

11) Vgl. Anhang 1 TEHG. 
oder zumindest ausgeglichen ( $\$ 11$ Abs. 2 BEHG) werden, sodass das BEHG vor allem in den Sektoren Gebäudewärme und Verkehr Relevanz entfaltet. ${ }^{12}$

\subsection{Funktionsweise und Preisbildung}

Um den marktbasierten Anreiz zur Emissionsminderung zu setzen, wird ein Grenzwert festgelegt, unter dem die jährlichen Gesamtemissionen, die aus der Verbrennung der erfassten Stoffe resultieren, bleiben müssen (sog. jährliche Emissionsmenge, \$4 BEHG). Die Festlegung erfolgt nach $\int 4$ Abs. 1 BEHG für jedes Kalenderjahr innerhalb einer Handelsperiode und muss die Einhaltung der Minderungspflichten der Bundesrepublik Deutschland nach Art. 4 Abs. 1 i.V.m. Anhang 1 der EU-Klimaschutzverordnung gewährleisten.

Die Verantwortlichen, ${ }^{13}$ d. h. diejenigen natürlichen oder juristischen Personen, die die Brennstoffe entsprechend den energiesteuerlichen Tatbeständen in den Verkehr bringen ( $\$ \$ 3$ Nr. 3, 2 Abs. 2 BEHG), müssen eine entsprechende Menge Zertifikate für das Inverkehrbringen nachweisen ( $\$ 8$ BEHG). Diese können sie entweder im Zuge einer Versteigerung durch das Umweltbundesamt (SS 10,13 Abs. 1 BEHG) oder auf dem nachgelagerten freien Sekundärmarkt ( $\int 9$ Abs. 2 BEHG) erwerben. Die Kombination aus der Pflicht, Zertifikate abzugeben, und der Tatsache, dass diese zumindest im Ursprung vom Staat erworben werden müssen, begründet eine Geldleistungspflicht der Bürger an den Staat und stellt somit eine öffentlich-rechtliche Abgabe dar. ${ }^{14}$ Nach dem ökonomischen Grundgedanken werden die Verantwortlichen das dadurch entstehende Preissignal an die Endverbraucher der Brennstoffe, d. h. die Emittenten, weitergeben. ${ }^{15}$ Diese werden Emissionsminderungsmaßnahmen wahrnehmen, wenn diese kostengünstiger sind als der Kauf von Zertifikaten. Damit verlagern sich Einsparmaßnahmen automatisch an die aus gesamtgesellschaftlicher Sicht kosteneffizienteste Stelle.

Die Bepreisung soll zunächst im Zeitraum 2021 bis 2025 durch einen sich jährlich anhebenden Fixpreis erfolgen (Preispfad), der dann 2026 durch einen Preiskorridor, d.h. einen Mindest- und Höchstpreis, abgelöst wird. Eine vollkommen freie Preisbildung am Markt soll - vorbehaltlich einer Entscheidung zur Fortführung des Preiskorridors im Jahr 2025 (\$23 Abs. 1 S. 4 u. 5 BEHG) - erst ab dem Jahr 2027 stattfinden. Nach der ursprünglichen Fassung des BEHG betrug der Startpreis für das Jahr 202110 Euro und steigt auf 20 Euro im Folgejahr und danach jährlich um 5 Euro an, sodass er 2025 bei 35 Euro liegt. Der Preiskorridor sollte im Jahr 2026 bei 35-60 Euro liegen ( $\int 10$ Abs. 2 S. 4 BEHG). Der geringe Einstiegspreis sowie die niedrige Preisentwicklung bis 2027 wurden allerdings bereits bei der Verbändeanhörung im Gesetzgebungsverfahren als ineffektiv und ungenügend kritisiert. ${ }^{16} \mathrm{Nach}$ den politischen Vereinbarungen im Vermittlungsausschuss, der durch den Bundesrat zum „,Gesetz zur Umsetzung des Klimaschutzprogramms $2030 \mathrm{im}$ Steuerrecht" ${ }^{17}$ angerufen wurde, ${ }^{18}$ wurde eine erste Gesetzesänderung des BEHG beschlossen, die den Startpreis auf 25 Euro anhebt. Der Fixpreis steigt nun jährlich bis 2023 um 5 Euro und bis 2025 um 10 Euro pro Jahr an. Der Preiskorridor für das Jahr 2026 liegt bei 55-65 Euro. ${ }^{19}$

Mit der anfänglichen Preisfixierung sowie der Einführung eines Mindest- und Höchstpreises im Jahr 2026 sollen Unsicherheiten bezüglich des Preisniveaus vermieden und somit die Investitionssicherheit gesteigert werden. ${ }^{20}$ Um sicherzustellen, dass durch einen überhöhten Kauf von Zertifikaten im Einführungszeitraum bis 2025 keine Überschreitung der jährlichen Emissionsmengen im Folgezeitraum droht, sind in der Einführungsphase gekaufte Zertifikate nur für die Abdeckung der Brennstoffemissionen des entsprechenden Kalender- oder Vorjahrs gültig ( $\$ 9$ Abs. 1 S. 3 BEHG).
Da für den Zeitraum 2021 bis 2025 ein Fixpreis und für das Folgejahr eine Preisobergrenze vorgesehen sind und diese Ausgestaltungsoptionen nur durch eine Ausgabe von Zertifikaten im vollen Umfang der Nachfrage erreicht werden können, kann die mengenmäßige Begrenzung durch das BEHG selbst nicht garantiert werden. \5 BEHG sieht für diese Zeit die Nutzung von Flexibilisierungsinstrumenten nach der EU-Klimaschutzverordnung ${ }^{21}$ vor, die unter anderem den Zukauf zusätzlicher Emissionszuweisungen von anderen Mitgliedstaaten umfassen.

\section{Kann der Zertifikatekauf als Steuer eingeordnet werden?}

Bei Einführung des BEHG wurden Stimmen laut, nach deren Auffassung das Gesetz in der Anfangszeit bis 2027 in Wahrheit eine Steuer darstelle 22 oder zumindest in der Wirkungsweise mit einer solchen vergleichbar $\mathrm{sei}^{23}$. Be-

12) BT-Drs. 19/14746, S. 20.

13) In diesem Aufsatz wurden nach Möglichkeit genderneutrale Formulierungen gewählt. Wo dies nicht möglich war, wurde aus Gründen der Vereinheitlichung innerhalb der Zeitschrift auf das generische Maskulinum zurückgegriffen. Ausdrücklich sind hiermit alle Geschlechter angesprochen.

14) So für die Bestimmung der Abgabenqualität im EU-ETS: BVerwG, Urt. v. 10. 10. 2012 - 7 C 10/10, NVwZ 2013, 576, 580; das BVerfG spricht insofern von einer ,,abgabengleichen Wirkung“": Beschl. v. 5.3.2018 - 1 BvR 2864/13, juris, Rdnr. 31; vgl. auch: Wernsmann/Bering, NVwZ 2020, 497, 498.

15) Grundlegend zur Idee des Zertifikatehandels: Coase, The Journal of Law \& Economics, 1960, Bd. 3, 1; vgl. auch Sturm/Vogt, Umweltökonomik, 2. Aufl. 2018, S. 90 ff.; zusammenfassend: Edenhofer/Flachsland/Kalkuhl/Knopf/Pahle, Optionen für eine $\mathrm{CO}_{2}-$ Preisreform, MCC-PIK-Expertise für den Sachverständigenrat zur Begutachtung der gesamtwirtschaftlichen Situation, 2019, S. $22 \mathrm{ff}$.

16) Siehe z.B.: Bundesverband Erneuerbare Energien e.V., Stellungnahme zum Referentenentwurf des Bundesministeriums für Umwelt, Naturschutz und nukleare Sicherheit zum Entwurf eines Gesetzes über ein nationales Emissionshandelssystem für Brennstoffemissionen, 19.10.2019, S. 4f.; Greenpeace, Stellungnahme zum Entwurf eines Gesetzes über ein nationales Emissionshandelssystem für Brennstoffemissionen, 21.10.2019, S. 2; demgegenüber bewertet der DIHK die Preisausgestaltung aus Sicht der Wirtschaft positiv: DIHK Stellungnahme BEHGE, 4.11.2019, S. 2f.; siehe auch: Edenhofer/Flachsland/Kalkuhl/ Knopf/Pahle, Bewertung des Klimapakets und nächste Schritte: $\mathrm{CO}_{2}$-Preis, sozialer Ausgleich, Europa, Monitoring, MCC-PIK, 2019, S. 4 ff.; IKEM/Rodi, Verfassungsmäßigkeit des Entwurfs zum Brennstoffemissionshandelsgesetz (BEHG-E), November 2019, S. 8f. mit Verweis auf: The Boston Consulting Group/prognos, Klimapfade für Deutschland, Januar 2018, S. 77 ff.; einen Überblick über relevante $\mathrm{CO}_{2}$-Preisniveaus gibt: Matthes, Der Preis auf $\mathrm{CO}_{2}$, Heinrich Böll Stiftung - Schriften zur Ökologie, Bd. 48, S. $30 \mathrm{ff}$.

17) Gesetz zur Umsetzung des Klimaschutzprogramms $2030 \mathrm{im}$ Steuerrecht v. 21.12.2019, BGB1. I S. 2886.

18) Vgl. BR-Drs. 608/19(B).

19) Bei Bearbeitungsende des Aufsatzes wurde die Gesetzesveränderung noch nicht im Bundesgesetzblatt veröffentlicht. Zur dritten Beratung und Schlussabstimmung siehe BT-P1Pr. 19/183, S. $22971 \mathrm{ff}$. Zum Gesetzesentwurf siehe BT-Drs. 19/19929, zur Beschlussempfehlung des Ausschusses für Umwelt, Naturschutz und nukleare Sicherheit BT-Drs. 19/23184.

20) BT-Drs. 19/14746, S. 37.

21) Siehe Art. 5 VO (EU) 2018/842.

22) Steinbach/Valta, JZ 2019, 1139, $1142 \mathrm{f}$

23) IKEM/ Rodi, Verfassungsmäßigkeit des Entwurfs zum Brennstoffemissionshandelsgesetz (BEHG-E), November 2019, S. 13; Müller/Kahl, Zur verfassungsrechtlichen Einordnung des Brennstoffemissionshandelsgesetzes - Stellungnahme zur Anhörung des Ausschusses für Umwelt, Naturschutz und nukleare Sicherheit im Deutschen Bundestag am 6.11.2019, Würzburger Berichte zum Umweltenergierecht, Nr. 45, 4.11.2019, S. $12 \mathrm{ff}$; Wünnemann, DStR 2019, 2099. 
gründet wurde dies mit dem Gedanken, dass in dieser Zeit keine mengenmäßige Begrenzung der Emissionen vorläge und das Instrument deshalb preisbasiert wie eine Steuer und nicht mengenbasiert wie ein Zertifikatehandel wirke. Eine solche Einordnung setzt allerdings voraus, dass das Instrument unter die hergebrachte Definition der Steuer subsumiert werden kann. Die Einordnung ist für die Bestimmung der Kompetenzgrundlage relevant. Während Steuern den Kompetenzverteilungen und Zulässigkeitsvoraussetzungen der Finanzverfassung in den Artt. 104a ff. GG unterfallen, gelten für nichtsteuerliche Abgaben die allgemeinen Sachgesetzgebungskompetenzregelungen der Artt. 70 ff. GG. ${ }^{24}$ Für die Bestimmung ist dabei nicht die Bezeichnung, sondern vielmehr der materielle Gehalt der Abgabe von Bedeutung. ${ }^{25}$

Nach der etablierten Definition handelt es sich bei Steuern im verfassungsrechtlichen Sinn um ,öffentliche Abgaben, die als Gemeinlast ohne individuelle Gegenleistung (,,voraussetzungslos") zur Deckung des allgemeinen Finanzbedarfs eines öffentlichen Gemeinwesens erhoben werden“". ${ }^{26}$

Dem Kriterium der Einnahmenerzielung steht nicht entgegen, dass vorrangig ein Lenkungszweck verfolgt wird, solange die Einnahmenerzielung zumindest Nebenzweck bleibt. ${ }^{27}$ Deshalb erweist sich das Kriterium der Gegenleistungslosigkeit als geeigneteres Abgrenzungsmerkmal, um Steuern von sonstigen Abgaben unterscheiden zu können. Nach Feststellung des BVerfG zum EU-ETS ist die Gegenleistung beim dortigen Emissionshandel in den erworbenen Emissionsberechtigungen zu sehen. ${ }^{28}$ Angewendet auf das BEHG, das nicht bei den Emissionen direkt, sondern bei dem Inverkehrbringen der Brennstoffe anknüpft, bedeutet dies, dass die Gegenleistung in der Berechtigung zum Inverkehrbringen der erfassten Brennstoffe liegt, die durch das Zertifikat abgebildet wird. ${ }^{29}$ Warum diese Bewertung in der Einführungsphase anders ausfallen sollte, nur weil die Anzahl der ausgegebenen Zertifikate nicht begrenzt ist, ist nicht ersichtlich. Die Pflicht nach $\$ 8$ BEHG zum Nachweis einer entsprechenden Menge an Zertifikaten für das Inverkehrbringen von Brennstoffen besteht nämlich auch in dieser Phase unverändert. ${ }^{30}$

Somit scheitert die Einordnung des BEHG als Steuer auch in der Anfangsphase an dem Vorliegen einer Gegenleistung, da auch hier bereits Zertifikate ausgegeben werden. Als Kompetenzgrundlage ist somit eine Sachkompetenz aus den Artt. 70 ff. GG erforderlich. Auf materiell-rechtlicher Seite bestehen die besonderen Rechtfertigungserfordernisse, die an nichtsteuerliche Abgaben zu stellen sind.

\section{Der Zertifikatserwerb als nichtsteuerliche Abgabe}

\subsection{Einordnung als Vorzugslast oder Sonderabgabe}

Im Grundgesetz sind die nichtsteuerlichen Abgaben nicht abschließend benannt. ${ }^{31}$ Der Legislative steht es somit grundsätzlich offen, Abgaben auf ihre Sachgesetzgebungskompetenz aus den Artt. 70 ff. GG zu stützen. ${ }^{32}$ Bei ,,typisierender Betrachtung " ${ }^{33}$ hat die Rechtsprechung allerdings durchaus verschiedene Arten von nichtsteuerlichen Abgaben hervorgebracht. Neben den sog. Vorzugslasten, die jedenfalls Gebühren und Beiträge erfassen, sind zumindest die Anforderungen an Sonderabgaben im engeren Sinn ${ }^{34}$ von der Rechtsprechung recht klar umrissen worden.

Gebühren sind ,öffentlich-rechtliche Geldleistungen ..., die aus Anlass individuell zurechenbarer Leistungen dem Gebührenschuldner durch eine öffentlich-rechtliche Norm oder sonstige hoheitliche Maßnahme auferlegt werden“. 35 Beiträge werden hingegen bereits für die ,potentielle Inanspruchnahme einer öffentlichen Einrichtung oder Leistung erhoben“. ${ }^{36}$ Die Rechtfertigung dieser Vorzugslasten ist regelmäßig unproblematisch ${ }^{37}$ und ergibt sich aus der Tatsache, dass ein staatlicher Kostenaufwand ausgeglichen werden soll. ${ }^{38} \mathrm{Da}$ die Zertifikate nur im Umfang der tatsächlich in Verkehr gebrachten Menge an Brennstoffen abgegeben werden müssen, kommt allenfalls eine Gebühr in Betracht. Einer solchen Einordnung steht aber entgegen, dass kein staatlicher Kostenaufwand besteht, der mit der Gebühr ausgeglichen werden soll. Die Geldleistungspflicht bei den Emissionszertifikaten ergibt sich aus der umweltökonomischen Überlegung, dass durch ein Preissignal die Externalität „Umweltbelastung“ in der Emissionsentscheidung eingepreist werden soll, und nicht etwa aus dem damit verbundenen Verwaltungsaufwand, für den ergänzende Gebührenregelungen bestehen ( $\left(16\right.$ BEHG) ${ }^{39}$

Da die nichtsteuerlichen Abgabenarten „Gebühr“ und „Beitrag“ aufgrund des fehlenden staatlichen Kostenaufwands nicht in Betracht kommen, ist nur noch die Einordnung als Sonderabgabe oder sonstige Abgabe sui generis ${ }^{40}$ möglich.

\subsection{Sonderabgaben im engeren und weiteren Sinn}

Bei der Einordnung als Sonderabgabe ist zunächst zwischen Sonderabgaben im engeren und weiteren Sinn zu unterscheiden. ${ }^{41}$

24) Heun, in: Dreier, Grundgesetz-Kommentar, 3. Aufl. 2018 , Art. 105 Rdnr. 18; Seiler, in: Maunz/Dürig, Grundgesetz-Kommentar, Stand: 89. EL Oktober 2019, Art. 105 Rdnr. 80.

25) In st. Rspr. BVerfG, Beschl. v. 7. 11.1995 - 2 BvR 413/88, 2 BvR 1300/93, BVerfGE 93, 319, 345, mit Verweis auf: BVerfG, Beschl. v. 6.2.1979 - 2 BvL 5/76, BVerfGE 50, 217, 225 f.; außer Betracht bleibt daher die ausdrückliche Nennung der Kompetenzgrundlage des Art. 74 Abs. 1 Nr. 24 und 11 GG in der Gesetzesbegründung: BT-Drs. 19/14746, S. 22.

26) BVerfG, Urt. v. 20.4.2004 - 1 BvR 1748/99, 1 BvR 905/00, BVerfGE 110, 274, 294f.; BVerfG, Beschl. v. 13.4.2017 - 2 BvL 6/13, juris, Rdnr. 100.

27) BVerfG, Beschl. v. 22.6.1995 - 2 BvL 37/91, BVerfGE 93, 121, 147 f.; BVerwG, Beschl. v. 19.8. 1994 - 8 N 1/93; NVwZ 1995 , 59, 61; Jachmann-Michel/Vogel, in: v. Mangoldt/Klein/Starck, Grundgesetz-Kommentar, 7. Aufl. 2018, Art. 105 Rdnr. 4; Seiler, in: Maunz/Dürig, Grundgesetz-Kommentar, Stand: 89. EL Oktober 2019, Art. 105 Rdnr. 38

28) BVerfG, Nichtannahmebeschl. v. 5.3.2018 - 1 BvR 2864/13, juris, Rdnr. 26.

29) So auch: Klinski/Keimeyer, ZUR 2020, 342, 346.

30) Steinbach/Valta sehen dahingegen in der Einführungsphase keine Gegenleistung in den Zertifikaten, die sie als reine Zahlungsbelege begreifen: JZ 2019, 1139, $1142 \mathrm{f}$.

31) BVerfG, Beschl. v. 7. 11.1995 - 2 BvR 413/88, 1300/93, BVerfGE 93, 319, 345; Jachmann-Michel/Vogel, in: v. Mangoldt/Klein/Starck, Grundgesetz-Kommentar, 7. Aufl. 2018, Art. 105 Rdnr. 8.

32) Seiler, in: Maunz/Dürig, Grundgesetz-Kommentar, 74. EL Mai 2015, Art. 105 Rdnr. 80.

33) Seiler, in: Maunz/Dürig, Grundgesetz-Kommentar, 74. EL Mai 2015, Art. 105 Rdnr. 80.

34) BVerfG, Beschl. v. 17.7.2003 - 2 BvL 1, 4, 6, 16, 18/99, 1/01, BVerfGE 108, 186, $216 \mathrm{ff}$.

35) BVerfG, Urt. v. 18.7.2018 - 1 BvR 1675/16, 745, 836, 981/17, BVerfGE 149, 222, 250.

36) BVerfG, Urt. v. 18.7.2018 - 1 BvR 1675/16, 745, 836, 981/17, BVerfGE 149, 222, 250.

37) BVerfG, Beschl. v. 7. 11. 1995-2 BvR 413/88, 1300/93, BVerfGE 93, 319, $343 \mathrm{f}$.

38) BVerfG, Beschl .v. 9.11.1999 - 2 BvL 5/95, BVerfGE 101, 141, 148.

39) Ausführlich dazu: Wernsmann/Bering, NVwZ 2020, 497, $500 \mathrm{f}$.

40) Sonstige Abgaben sui generis sind beispielsweise Verbandslasten, Sozialversicherungs- und Rundfunkbeiträge, vgl. Jachmann-Michel/Vogel, in: v. Mangoldt/Klein/Starck, Grundgesetz-Kommentar, 7 Aufl. 2018, Art. 105 Rdnr. 15.

41) BVerfG, Urt. v. 26.5.1981 - 1 BvL 56, 57, 58/78, BVerfGE 57, 139, 167 f.; BVerfG, Urt. v. 6. 11.1984 - 2 BvL 19, 20/83, 2 BvR 363, 491/83, BVerfGE 67, 256, 274 ff.; BVerfG, Nichtannahmebeschl. v. 5.3.2009 - 2 BvR 1824/05, juris, Rdnr. $24 \mathrm{ff}$; vgl. auch: Elsner/Kaltenborn, JA 2005, 823, 826, m. w. N. 
Erstere werden ohne Gegenleistung erhoben und weisen somit eine Finanzierungsfunktion auf, die sie in die Nähe einer Steuer rückt. Für diese Kategorie bestehen erhöhte Rechtfertigungsanforderungen, wobei sich die Rechtfertigung insbesondere nicht allein aus dem Zweck der Einnahmenerzielung ergeben kann. ${ }^{42}$ Entscheidend ist allein der materielle Zusammenhang der Abgabe mit dem angestrebten Zweck, ${ }^{43}$ der auf einer entsprechenden Sachkompetenz aus den Artt. 70 ff. GG fußen muss. ${ }^{44}$ Dieses besondere Rechtfertigungsbedürfnis ergibt sich aus dem „Prinzip des Steuerstaates" ${ }^{45}$ nachdem die Haushaltshoheit des Parlaments und der Regierung grundsätzlich dadurch zu garantieren ist, dass keine „Einnahme- und Ausgabekreisläufe außerhalb des Budgets" geschaffen werden. ${ }^{46}$ Den Regelungen der Artt. 104a ff. GG wird eine Grundsatzentscheidung der Verfassung zur Finanzierung des Haushalts durch Steuern als Regeltypus entnommen. ${ }^{47}$ Neben der föderativ motivierten Funktion der Artt. 104a ff. GG, eine klare Kompetenzverteilung sowohl hinsichtlich der Aufgabe der Steuererhebung als auch der Verteilung der Einnahmen und Ausgaben zwischen Bund und Ländern vorzunehmen, ${ }^{48}$ dienen die Bestimmungen auch dem Schutz der Bürger vor einer übermäßigen Abgabenlast (sog. Schutzund Begrenzungsfunktion). ${ }^{49}$ Die Erhebung nichtsteuerlicher Abgaben bleibt dem Staat daher nicht verwehrt, unterliegt jedoch strengen Zulässigkeitskriterien ${ }^{50}$ und einem erhöhten Rechtfertigungserfordernis ${ }^{51}$. Dies soll sicherstellen, dass die Voraussetzungen für die Steuererhebung nicht umgangen werden.

Demgegenüber verfolgen Sonderabgaben im weiteren Sinn keine Finanzierungs-, sondern eine Lenkungsfunktion. ${ }^{52}$ Durch das Setzen wirtschaftlicher Anreize soll Einfluss auf das Verhalten der Abgabenschuldner genommen und somit der Sachzweck erreicht werden. ${ }^{53}$ Da die Distanz zu einer Steuer hier größer ist, sind sie leichter zu rechtfertigen.$^{54}$ Dies schlägt sich auch bei den Zulässigkeitskriterien nieder, die nach dem BVerfG für Sonderabgaben im weiteren Sinn nur eingeschränkt gelten (s. u.). ${ }^{55}$

Wie gezeigt, setzt das BEHG mit der Kappung der zulässigen Gesamtemissionen und der Ausgabe kostenpflichtiger Zertifikate ein Preissignal, das zu einer Emissionsminderung bei den Endverbrauchern der Brennstoffe führen soll. Es verfolgt somit einen Lenkungszweck. Diese Feststellung wird durch das Bestehen einer Gegenleistung in Form der Berechtigung zum Inverkehrbringen der Brennstoffe unterstützt, die die Abgrenzung zur Steuer unterstreicht. ${ }^{56}$ Voraussetzung für die Einordnung als Lenkungsabgabe ist, dass die Sonderabgabe keinerlei Finanzierungszweck, nicht einmal als Nebenzweck, verfolgt. ${ }^{57}$ Da Abgaben notwendigerweise Einnahmen generieren, ist zur Abgrenzung darauf abzustellen, ob die Abgabe ,vollständig vom Lenkungszweck geprägt ist“; die Lenkungsfunktion dem Finanzierungseffekt der Abgabe also ,funktional zuwiderläuft". ${ }^{8}$ Beim Emissionshandel sollen die Emissionen von Treibhausgasen möglichst weit zurückgehen, indem die Endverbraucher ihr Verhalten umstellen. Funktioniert das System, werden weniger Zertifikate gekauft, weil emissionsarme Technologien preiswerter werden. Das Ziel des BEHG ist also dann erreicht, wenn keine Einnahmen durch die Versteigerung von Emissionszertifikaten mehr erzielt werden, sodass dem BEHG eine reine Lenkungsfunktion zukommt. Die Mittelverwendung zum Aufbau eines Handelssystems, das immanenter Bestandteil des ökonomischen Instruments ,Zertifikatehandel“" und damit wesentlich für dessen Funktionsweise ist, ändert nichts an dem Vorliegen einer reinen Lenkungsfunktion. Das BVerfG hat in dem Nichtannahmebeschluss zum Zuteilungsgesetz 2012 $(\mathrm{ZuG} 2012)^{59}$ keine Bedenken an der Tatsache geäußert, dass die nach $\$ 19 \mathrm{ZuG} 2012$ erzielten Einnahmen für den Aufbau des „Gemeinschaftssystems für den Handel mit Treibhausgasemissionszertifikaten" vorgesehen waren und ausdrücklich festgestellt, dass solche Veräußerungsentgelte nicht unter ,die finanzverfassungsrechtlichen Bestimmungen über Finanzmonopole und Steuern" fallen. ${ }^{60}$ Die im BEHG vorgesehene Erlösverwendung für die durch das BEHG entstehenden Aufgaben, d.h. dem Aufbau und der Durchführung eines entsprechenden Handelssystems, folgt vergleichbaren Maßstäben.

Das unter dem BEHG geschaffene System zum Kauf und Nachweis von Zertifikaten ist somit als Sonderabgabe im

42) BVerfG, Nichtannahmebeschl. v. 5.3.2018-1 BvR 2864/13, juris, Rdnr. 30, m.w. N.

43) Seiler, in: Maunz/Dürig, Grundgesetz-Kommentar, 74. EL Mai 2015, Art. 105 Rdnr. 80, mit Verweis auf: BVerfG, Urt. v. 6.11.1984 - 2 BvL 19, 20/83, 2 BvR 363, 491/83, BVerfGE 67, 256, 275 f.; vgl. auch: BVerwG, Urt. v. 22.11.2000 - 6 C 8/99, BVerwGE 112, 194, juris, Rdnr. 20, mit Verweis auf: BVerfG, Beschl. v. 4.2.1958 - 2 BvL 31, 33/56, BVerfGE 7, 244, 252; BVerfG, Beschl. v. 12.10.1978 - 2 BvR 154/74, BVerfGE 49, 343, 353; BVerfG, Urt. v. 10.12.1980 - 2 BvF 3/77, BVerfGE 55, 274, 305; BVerfG, Beschl. v. 24.1.1995 - 1 BvL 18/93, 5, 6, 7/94, 1 BvR 403, 569/94, BVerfGE 92, 91, 114.

44) BVerfG, Urt. v. 10.12.1980 - 2 BvF 3/77, BVerfGE 55, 274, $304 \mathrm{f}$.

45) BVerfG, Beschl. v. 7.11.1995-2 BvR 413/88, 1300/93, BVerfGE 93, 319, 342, mit Verweis auf: BVerfG, Beschl. v. 8.6.19882 BvL 9/85, 3/86, BVerfGE 78, 249, 266f.; BVerfG, Beschl. v. 31.5. 1990 - 2 BvL 12, 13/88, 2 BvR 1436/87, BVerfGE 82, 159 , $178 \mathrm{f}$.

46) BVerfG, Beschl. v. 7.11.1995 - 2 BvR 413/88, 1300/93, BVerfGE 93, 319, 343, mit Verweis auf: BVerfG, Beschl. v. 31.5.1990 2 BvL 12, 13/88, 2 BvR 1436/87, BVerfGE 82, 159, 179; BVerfG, Beschl. v. 11.10.1994 - 2 BvR 633/86, BVerfGE 91, 186, 202.

47) Heun, in: Dreier, Grundgesetz-Kommentar, 3. Aufl. 2018, Art. 105 Rdnr. 11; Seiler, in: Maunz/Dürig, Grundgesetz-Kommentar, 74. EL Mai 2015, Art. 105 Rdnr. 41.

48) BVerfG, Beschl. v. 13.4.2017 - 2 BvL 6/13, BVerfGE 145, 171, $190 f .$, m.w. N.

49) BVerfG, Beschl. v. 31.5.1990 - 2 BvL 12, 13/88, 2 BvR 1436/87, BVerfGE 82, 159, 179; BVerfG, Beschl. v. 11.10.1994-2 BvR 633/8, BVerfGE 91, 186, 202 f.; BVerfG, Beschl. v. 7.11.1995 2 BvR 413/88, 1300/93, BVerfGE 93, 319, 342; BVerfG, Besch1. v. 13. 4. $2017-2$ BvL 6/13, BVerfGE 145, 171, $191 \mathrm{f} ., 203 \mathrm{f}$.

50) BVerfG, Beschl. v. 31.5.1990 - 2 BvL 12, 13/88, 2 BvR 1436/87, BVerfGE 82, 159, 179, mit Verweis auf: BVerfG, Urt. v. 10.12. $1980-2$ BvF 3/77, BVerfGE 55, 274, 298; BVerfG, Urt. v. 6.11.1984-2 BvL 19, 20/83, 2 BvR 363, 491/83, BVerfGE 67, 256, $275 \mathrm{f}$.

51) In st. Rspr. BVerfG, Beschl. v. 13.4.2017 - 2 BvL 6/13, juris, Rdnr. 102.

52) BVerfG, Urt. v. 6.11.1984 - 2 BvL 19, 20/83, 2 BvR 363 , 491/83, BVerfGE 67, 256, 277 f.; BVerfG, Nichtannahmebeschl. v. 5.3.2009-2 BvR 1824/05, juris, Rdnr. 25

53) Elsner/Kaltenborn, JA 2005, 823, 826.

54) BVerfG, Beschl. v. 8.6.1988 - 2 BvL 9/85, 2 BvL 3/86, NJW 1988, 2529, 2530; Osterloh, NVwZ 1991, 823, 825.

55) BVerfG, Urt. v. 26.5.1981 - 1 BvL 56, 57, 58/78, BVerfGE 57, 139, 167f.; BVerfG, Urt. v. 6.11.1984-2 BvL 19, 20/83, 2 BvR 363, 491/83, BVerfGE 67, 256, 277 f.; BVerfG, Nichtannahmebeschl. v. 5.3.2009 - 2 BvR 1824/05, juris, Rdnr. 25; Heintzen, in: v. Münch/Kunig, Grundgesetz-Kommentar, 6. Aufl. 2012, Art. 105 Rdnr. 27; dazu sogleich.

56) $\mathrm{Zu}$ den erleichterten Zulässigkeitsvoraussetzungen einer Sonderabgabe, wenn sie nicht ,in Konkurrenz zu den Steuern tritt“: BVerfG, Beschl. v. 5.3.2009 - 2 BvR 1824/05, juris, Rdnr. 25.

57) BVerfG, Urt. v. 6. 11.1984-2 BvL 19, 20/83, 2 BvR 363, 491/83, BVerfGE 67, 256, 278.

58) Jachmann, NVwZ 1992, 932, 933; vgl. auch: Jachmann-Michel/ Vogel, in: v. Mangoldt/Klein/Starck, Grundgesetz-Kommentar, 7. Aufl. 2018, Art. 105, Rdnr. 17, mit Verweis auf: Elsner/Kaltenborn, JA 2005, 823, 826.

59) Gesetz zur Änderung der Rechtsgrundlagen zum Emissionshandel im Hinblick auf die Zuteilungsperiode 2008 bis $2012 \mathrm{v}$. 7.8.2007, BGB1. I S. 1788; zuletzt geändert durch Art. 4 Absatz 32 des Gesetzes v. 18. 6.2016, BGBl. I S. 1666.

60) BVerfG, Nichtannahmebeschl. v. 5.3.2018-1 BvR 2864/13, juris, Rdnr. 25 
weiteren Sinn einzuordnen, da es eine reine Lenkungsfunktion verfolgt.

\subsection{Zulässigkeitsvoraussetzungen der Sonderabgabe im weiteren Sinn}

Auch wenn eine (grund)gesetzliche Definition der Sonderabgabe nicht besteht, so hat die Rechtsprechung diesen $\mathrm{Ab}-$ gabentypus durch die richterrechtliche Verfestigung seiner Voraussetzungen doch klar umrissen. ${ }^{61}$ Wie bereits angesprochen, muss der Gesetzgeber mit ihr einen Sachzweck verfolgen, der über die reine Mittelbeschaffung hinausgeht. Emissionshandelssysteme können entsprechend der Bewertung des BVerfG im Nichtannahmebeschluss zum ZuG 2012 auf die konkurrierende Gesetzgebungskompetenz des Bundes zur Luftreinhaltung auf Art. 74 Abs. 1 Nr. 24 GG gestützt werden. ${ }^{62}$ Auf diesen wurde sich auch bei der Einführung des BEHG bezogen, ${ }^{63}$ sodass offensichtlich wird, dass das Gesetz den Sachzweck der Emissionsminderung verfolgt

In der jüngeren Zeit fordert das BVerfG zudem ,haushaltsrechtliche Informationspflichten“, um zu gewährleisten, dass die Haushaltshoheit des Parlaments nicht umgangen wird. Dafür ist erforderlich, dass ,alle erhobenen Sonderabgaben ... in einer Anlage zum Haushaltsplan vollständig dokumentiert werden. " ${ }^{64}$ Das BVerfG hat zum ZuG 2012 festgestellt, dass die Überführung der Einnahmen in den „Energie- und Klimafonds“ den haushaltsrechtlichen Informationspflichten zumindest nicht entgegensteht, wenn eine Dokumentation im Haushaltsgesetz erfolgt. ${ }^{65}$ Da auch die Einnahmen aus dem BEHG in den Sonderfonds geleitet werden $(\$ 4$ Abs. 1 Nr. 2 EKFG $),{ }^{66}$ ist auf eine entsprechende Dokumentation zu achten.

Sonderabgaben setzen, um klar von einer Steuer unterschieden werden zu können, voraus, dass nur eine von der Allgemeinheit abgrenzbare Gruppe durch die Abgabe belastet wird. Die Gruppe muss dabei aufgrund gemeinsamer Interessen oder anderer Gegebenheiten nach der gesellschaftlichen Wirklichkeit, die die Legislative aufgrund einer Kompetenznorm berücksichtigen durfte, abgrenzbar sein (sog. Gruppenhomogenität). ${ }^{67}$ Indem der Brennstoffemissionshandel sich auf die Inverkehrbringer der Brennstoffe nach der Energiesteuer bezieht, wird eine Gruppe zur Verantwortung gezogen, die eigene Interessen gegenüber der Allgemeinheit verfolgt und somit von ihr abgrenzbar ist. Betroffen sein sollen rund 4050 Unternehmen, ${ }^{68}$ sodass eine „Gruppenhomogenität" besteht.

Weiterhin muss die mit der Abgabe verfolgte Aufgabe grundsätzlich in den Verantwortungsbereich der Gruppe fallen (sog. Gruppenverantwortung kraft Sachnähe). ${ }^{69}$ In Abgrenzung zur Steuer muss die Einnahmenverwendung außerdem grundsätzlich zweckgebunden für die Aufgabenfinanzierung erfolgen und somit der Gruppe zugutekommen (sog. Gruppennützigkeit). ${ }^{70}$ Die Voraussetzungen der „Gruppenverantwortung“ und der ,gruppennützigen Verwendung" sind bei dem Zertifikatehandel, der eine Lenkungswirkung verfolgt, nicht vollständig erfüllt. Bereits an einer gruppennützigen Verwendung kann gezweifelt werden. Eine Begünstigung der Gruppe besteht zumindest insoweit, wie durch die Erlöse aus dem Verkauf der Zertifikate etwaige Beihilfen (vgl. \$11 BEHG) gedeckt werden sollen. ${ }^{71}$ Der Gruppennützigkeit steht insoweit nicht entgegen, dass einzelne Gruppenmitglieder nicht förderungsberechtigt sind. ${ }^{72} \mathrm{Ob}$ auch die Verwendung der Erlöse für die Kosten, die dem Bund durch die Wahrnehmung der ihm im BEHG zugewiesenen Aufgaben entstehen, d.h. den Aufbau und die Durchführung eines entsprechenden Handelssystems, zumindest mittelbar die Gruppe begünstigen, weil nur ein solches System den freien Handel mit den Zertifikaten und damit die Verlagerung der Emissionsminderung an die gesamtgesellschaft- lich kostengünstigste Stelle erlaubt, ist weniger offensichtlich. Für die Beurteilung ist zumindest unerheblich, dass auch Dritte (sonstige Personen) ein Konto zum Handel von Zertifikaten im nationalen Emissionshandelsregister einrichten können, ${ }^{73}$ denn eine sekundäre Begünstigung der Allgemeinheit lässt die ,gruppennützige Verwendung" nicht entfallen. ${ }^{74}$ Zumindest aber steht die Gruppe der erfassten Inverkehrbringer dem Zweck der Sicherstellung einer ,umweltschonenden, zuverlässigen und bezahlbaren Energieversorgung" sowie des Klimaschutzes (vgl. $\$ 2$ Abs. 1 EKFG), die durch den Energie- und Klimafonds finanziert werden sollen, nicht „,evident näher[...] als jede andere Gruppe oder die Allgemeinheit der Steuerzahler"75 Das Kriterium der „Gruppenverantwortung“ ist somit nicht erfüllt.

Die Voraussetzungen „Gruppenverantwortung“ und "Gruppennützige Verwendung" müssen aber bei Sonderabgaben mit Lenkungswirkung auch nicht uneingeschränkt erfüllt sein, da die Einnahmen bei Lenkungsabgaben nicht im Mittelpunkt stehen. ${ }^{76}$ Maßgeblich für die Verfassungskonformität ist, ob sich aus den ,speziellen Sach- und Zweckzusammenhängen“ ergebende „besondere Belastungsgründe" ${ }^{\text {"77 }}$ vorliegen, die die Sonderabgabe rechtfertigen können.

61) BVerfG, Urt. v. 10.12.1980 - 2 BvF 3/77, BVerfGE 55, 274 305 ff.; BVerfG, Urt. v. 6.11.1984 - 2 BvL 19, 20/83, 2 BvR 363, 491/83, BVerfGE 67, 256, 275 ff.; Heintzen, in: v. Münch/ Kunig, Grundgesetz-Kommentar, 6. Aufl. 2012, Art. 105 Rdnr. 25; Siekmann, in: Sachs, Grundgesetz-Kommentar, 8. Aufl. 2018, vor Art. 104a Rdnr. 152; Kube, in: Epping/Hillgruber, BeckOK Grundgesetz, 43. Ed. 15.5.2020, Art. 105 Rdnr. 17, m. w. N.

62) Vgl. BVerfG, Nichtannahmebeschl. v. 5.3.2018 - 1 BvR 2864/13, juris, Rdnr. 27.

63) BT-Drs. 19/14746, S. 37

64) Siekmann, in: Sachs, Grundgesetz-Kommentar, 8. Aufl. 2018, vor Art. 104a Rdnr. 165, mit Verweis auf: BVerfG, Beschl. v. 17.7.2003 - 2 BvL 1, 4, 6, 16, 18/99, 1/01, BVerfGE 108, 186 , $218 \mathrm{f}$.

65) BVerfG, Nichtannahmebeschl. v. 5.3.2018-1 BvR 2864/13, juris, Rdnr. 28

66) Gesetz zur Errichtung eines Sondervermögens „Energie- und Klimafonds“ v. 8.12.2010, BGB1. I S. 1807, zuletzt geändert durch Art. 3 des Gesetzes v. 12.12.2019, BGBl. I S. 2513.

67) BVerfG, Beschl. v. 5.3.1974 - 1 BvL 27/72, BVerfGE 37, 1, 16 BVerfG, Beschl. v. 31.5.1990 - 2 BvL 12, 13/88, 2 BvR 1436/87, BVerfGE 82, 159, 180; BVerfG, Beschl. v. 18.5.2004 - 2 BvR 2374/99, BVerfGE 110, 370, 389 f.; BVerfG, Urt. v. 10.12.1980 2 BvF 3/77, NJW 1981, 329, $333 \mathrm{f}$.

68) BT-Drs. $19 / 14746$, S. 8

69) BVerfG, Urt. v. 10.12.1980 - 2 BvF 3/77, BVerfGE 55, 274, 306 ; BVerfG, Beschl. v. 31.5.1990 - 2 BvL 12, 13/88, 2 BvR 1436/87, BVerfGE 82, 159, 180; BVerwG, Urt. v. 21.4.2004 - 6 C 20/03, NJW 2004, 3198, 3201.

70) BVerfG, Urt. v. 10.12.1980 - 2 BvF 3/77, BVerfGE 55, 274 $307 \mathrm{f}$.

71) Vgl. BT-Drs. 19/14746, S. 37.

72) BVerfG, Urt. v. 10.12.1980 - 2 BvF 3/77, BVerfGE 55, 274 $315 \mathrm{f}$.

73) Vgl. BT-Drs. 19/14746, S. 39.

74) Vgl. BVerfG, Urt. v. 10.12.1980 - 2 BvF 3/77, BVerfGE 55, 274, 317.

75) BVerfG, Urt. v. 28.1.2014 - 2 BvR 1561, 1562, 1563, 1564/12, BVerfGE 135, 155, 207.

76) BVerfG, Urt. v. 26.5.1981 - 1 BvL 56, 57, 58/78, BVerfGE 57, 139, 167; BVerfG, Urt. v. 6.11.1984- 2 BvL 19, 20/83, 2 BvR 363, 491/83, BVerfGE 67, 256, $277 \mathrm{f}$.

77) Jachmann-Michel/Vogel, in: v. Mangoldt/Klein/Starck, Grundgesetz-Kommentar, 7. Aufl. 2018, Art. 105 GG Rdnr. 20, mit Verweis auf: BVerfG, Urt. v. 26.5.1981 - 1 BvL 56, 57, 58/78, BVerfGE 57, 139, 167 f:; BVerfG, Urt. v. 6. 11.1984 - 2 BvL 19, 20/83, 2 BvR 363, 491/83, BVerfGE 67, 256, 277 f.; vgl. auch: BVerfG, Nichtannahmebeschl. v. 5.3.2009-2 BvR 1824/05, juris, Rdnr. 25. 


\subsection{Rechtfertigung der Sonderabgabe}

3.4.1 Rechtfertigung als Vorteilsabschöpfungsabgabe aufgrund einer mengenmäßigen Begrenzung

Zunächst kommt eine Rechtfertigung der Abgabe über den Grundgedanken der Vorteilsabschöpfung in Betracht. Der Belastungsgrund liegt hier in der Tatsache, dass ein Sondervorteil, den Einzelne durch die „bevorzugend gestattete Nutzung von Allgemeingütern" ${ }^{\text {"78 }}$ erhalten, abgeschöpft wird. Insofern liegt der Vorteilsabschöpfungsabgabe ein ähnlicher Ausgleichsgedanke wie der Gebühr zugrunde, nur bezieht sich der Ausgleich hier nicht auf einen staatlichen Kostenaufwand, sondern auf die Nutzung von Allgemeingütern. ${ }^{79}$

Das BVerfG hat in seinem Nichtannahmebeschluss zur Kürzung von Emissionsberechtigungen in Folge des $\mathrm{ZuG}$ 2012 die Abgabepflicht nach dem EU-ETS als eine Vorteilsabschöpfungsabgabe eingeordnet und die Vereinbarkeit mit der Finanzverfassung bejaht. ${ }^{80}$ Insofern erscheint auch die Einordnung des unter dem BEHG geschaffenen Systems als eine solche naheliegend. Zum EU-ETS hat das BVerfG ausgeführt, dass unter Beachtung des, ,weitreichenden Gestaltungsspielraum[s] “ der Legislative ${ }^{81}$ eine gerechtfertigte „Vorteilsabschöpfungsabgabe im Rahmen einer öffentlich-rechtlichen Nutzungsregelung "82 vorläge. Dem Nutzungsraum „Luft“ seien natürliche Ressourcengrenzen gesetzt, die die Bewirtschaftung durch ein Emissionshandelssystem i.S. e. Vorteilsabschöpfungsabgabe rechtfertigten. Voraussetzung hierfür sei allerdings, dass das „knappe Gut" durch die Bewirtschaftung auch eine tatsächliche mengenmäßige Begrenzung erfahre. Hierfür müsste die zulässige Emissionsmenge begrenzt werden, folglich weniger Zertifikate ausgegeben werden, als Nachfrage bestünde. Bei einer fehlenden Begrenzung käme es zu keiner Preisbildung und folglich zu keiner Lenkungswirkung, sodass das Instrument funktionslos sei. ${ }^{83}$

Auch im Bereich des BEHG wird durch ein Emissionshandelssystem der Nutzungsraum „Luft“ bewirtschaftet. Fraglich ist allein, ob auch eine mengenmäßige Begrenzung besteht. Auf lange Sicht kann dem BEHG eine solche nicht abgesprochen werden. Diese ergibt sich zum einen aus der bereits oben dargestellten Verpflichtung auf die Etappenziele der 55 \%igen Reduktion gegenüber 1990 bis 2030 und der Treibhausgasneutralität bis 2050, zum anderen aus den unter Berücksichtigung der EU-Klimaschutzverordnung festgesetzten jährlichen Emissionsmengen $(\$ 4$ Abs. 1 , 2 BEHG). Bedenken wurden nur in Bezug auf die Einführungs- und Übergangsphase bis 2027 geäußert. ${ }^{84} \mathrm{Da}$ in diesem Zeitraum ein Fixpreis bzw. eine Höchstpreis bestehe, der nur über eine unbegrenzte Zertifikatsausgabe erreicht werden könne, läge keine mengenmäßige Begrenzung vor, weswegen die Einordnung als Vorteilsabschöpfungsabgabe nicht möglich sei. Ein solches Verständnis setzt voraus, dass während der Eingangs- und Übergangsphase unbegrenzt Zertifikate ausgegeben werden, wenn der Fixpreis bzw. die Preisobergrenze erreicht ist. Dem BEHG lässt sich keine konkrete Aussage hierzu entnehmen. ${ }^{85}$

Unklar ist vor allem, ob Zertifikate über die ursprünglich gesetzte jährlichen Emissionsmengen hinaus unbegrenzt oder nur in dem Umfang ausgegeben werden können, in dem Flexibilisierungsinstrumente nach der EU-Klimaschutzverordnung 86 entsprechend $\$ 5$ BEHG genutzt werden können. ${ }^{87}$ Diese sehen auch den Zukauf von Emissionszuweisungen aus anderen Mitgliedsstaaten vor $(\$ 5$ Abs. $1 \mathrm{BEHG}$ ). Wäre die Mehrausgabe durch das Angebot der verfügbaren mitgliedstaatlichen Emissionszuweisung begrenzt, läge eine mengenmäßige Begrenzung vor. Für eine solche Lesart spricht der Wortlaut: $₫ 5$ Abs. 1 BEHG scheint von einer vollständigen Deckung der überschüssigen Emissionen durch die Flexibilisierungsinstrumente auszugehen. Dieser Betrachtung schließen sich auch der federführende Ausschuss für Umwelt, Naturschutz und nuk- leare Sicherheit und der Wirtschaftsausschuss des Bundesrates an, die anlässlich der Empfehlungen zum Entwurf des Ersten Gesetzes zur Änderung des BEHG feststellten, dass sich die Menge der Emissionszertifikate nach der EU-Klimaschutzverordnung richtet und durch die Nutzung der Flexibilisierungsinstrumente erhöht wird, die danach vorgegebene Höchstmenge aber nicht beliebig ausgeweitet werden könne. ${ }^{88}$ Insoweit spricht auch der Parl. Staatssekretär Pronold in der Antwort auf eine mündliche Frage im Bundestag von einer „hypothetischen Situation“ (in Bezug auf die Überschreitung der zulässigen Jahresemissionsmengen ohne möglichen Ausgleich durch die Flexibilisierungsinstrumente), und vertritt die Auffassung, dass die in $\ 5$ BEHG vorgesehenen Kompensationsmöglichkeiten ausreichen werden. ${ }^{89}$

$\mathrm{Ob}$ diese Auffassung zutreffend ist, wird die Praxis zeigen müssen. Aber auch wenn die erhöhte Ausgabe von Zertifikaten nicht durch die Flexibilisierungsinstrumente begrenzt sein sollte, ist nicht ersichtlich, warum die Einführungs- und Übergangsphase losgelöst von der Fortführung des Systems nach 2026 betrachtet wird. Vielmehr ist eine Gesamtbetrachtung des Emissionshandels auch über die Zeit nach 2026 hinaus angezeigt, weil die Menge der ausgegebenen Zertifikate im Zeitraum bis 2026 unmittelbar die jährlichen Emissionsmengen der Folgejahre beeinflusst. Die jährlichen Emissionsmengen werden unter Berücksichtigung des Emissionsdurchschnitts des fünften bis dritten Jahres vor Beginn der jeweiligen Handelsperiode berechnet ( $\$ 4$ Abs. 1 S. 2 BEHG). Durch den dortigen Verweis auf die EU-Klimaschutzverordnung ist zumindest indirekt auch der in Art. 4 Abs. 2 EU-Klimaschutzverordnung angesprochene lineare Minderungsweg erfasst. Sinnhaft ist die Minderungsverpflichtung nach der EU-Klimaschutzverordnung nur, wenn ein linearer Minderungspfad eingeschlagen wird, weil sich $\mathrm{CO}_{2}$-Emissionen für lange Dauer in der Atmosphäre einlagern ${ }^{90}$ und

78) Seiler, in: Maunz/Dürig, Grundgesetz-Kommentar, 74. EL Mai 2015, Art. 105 Rdnr. 86.

79) BVerfG, Beschl. v. 7.11.1995-2 BvR 413/88, 1300/93, BVerfGE 93, 319, $345 \mathrm{f}$.

80) BVerfG, Nichtannahmebeschl. v. 5.3.2018 - 1 BvR 2864/13, juris, Rdnr. $24 \mathrm{ff}$.

81) BVerfG, Nichtannahmebeschl. v. 5.3.2018 - 1 BvR 2864/13, juris, Rdnr. 30, mit Verweis auf: BVerfG, Beschl. v. 25.6.2014 1 BvR 668, 2104/10, BVerfGE 137, 1, 20 f., m. w. N.

82) BVerfG, Nichtannahmebeschl. v. 5.3.2018 - 1 BvR 2864/13, juris, Rdnr. $31 \mathrm{ff}$.

83) BVerfG, Nichtannahmebeschl. v. 5.3.2018-1 BvR 2864/13, juris, Rdnr. 35

84) Siehe z.B.: IKEM/Rodi, Verfassungsmäßigkeit des Entwurfs zum Brennstoffemissionshandelsgesetz (BEHG-E), November 2019 , S. $12 \mathrm{ff}$;; Müller/Kahl, Zur verfassungsrechtlichen Einordnung des Brennstoffemissionshandelsgesetzes - Stellungnahme zur Anhörung des Ausschusses für Umwelt, Naturschutz und nukleare Sicherheit im Deutschen Bundestag am 6.11.2019, Würzburger Berichte zum Umweltenergierecht, Nr. 45, 4.11.2019, S. 2 ff.; Wernsmann/Bering, NVwZ 2020, 497, $498 \mathrm{ff}$.; Klinski/Keimeyer, ZUR 2020, 342, $345 \mathrm{ff}$.

85) Da das Verhältnis von Fixpreis bzw. Höchstpreis zur Zertifikatsausgabe im BEHG unklar ist, war bereits im Gesetzgebungsverfahren eine Klarstellung empfohlen worden: vgl. Empfehlungen der Ausschüsse zur Stellungnahme des Bundesrates zum Gesetzentwurf nach Art. 76 Abs. 2 GG, BR-Drs. 533/1/19, S. 7.

86) Vgl. Artt. 5 u. 6 VO (EU) 2018/842.

87) Dazu ausführlich: Müller/Kahl, Zur verfassungsrechtlichen Einordnung des Brennstoffemissionshandelsgesetzes - Stellungnahme zur Anhörung des Ausschusses für Umwelt, Naturschutz und nukleare Sicherheit im Deutschen Bundestag am 6.11.2019, Würzburger Berichte zum Umweltenergierecht, Nr. 45, 4.11.2019, S. $5 \mathrm{ff}$

88) BR-Drs. 266/1/20, S. 2

89) PlPr. 19/169, S. 2111.

90) IPCC, Working group I contribution to the fifth assessment report, 2013, S. 472. 
deshalb kumulativ zu berücksichtigen sind. Das Erreichen des für 2030 festgesetzten Reduktionsziels um 38\% weniger gegenüber 2005 allein ist folglich nicht ausreichend; entscheidend ist auch der Weg dorthin. Wird in einzelnen Jahren der lineare Minderungspfad überschritten, so müssen in den Folgejahren mehr Emissionen eingespart werden, um die durch den linearen Minderungspfad vorgegebene Gesamtmenge nicht zu überschreiten. Gründe dafür, dass von diesem linearen Minderungspfad nach Vorstellung des Gesetzgebers abgewichen werden sollte, lassen sich der Gesetzesbegründung nicht entnehmen. Vielmehr wird der Einsatz von Flexibilisierungsinstrumenten in der Einführungsphase innerhalb der „,festgelegten Emissionsobergrenzen" erwogen ${ }^{91}$ und in $\$ 5$ Abs. 1 BEHG, der auf die ,Jahresmengen der EU-Klimaschutzverordnung" verweist, auch vorgesehen. Dies spricht dafür, dass sich das BEHG in die unionsrechtliche Systematik einfügen soll. Werden die Zielvorgaben in der Anfangszeit durch eine übermäßige Ausgabe von Zertifikaten überschritten und erfolgt kein Ausgleich über die Flexibilisierungsmaßnahmen nach $₫ 5$ BEHG, muss eine verstärkte Minderung in den Folgejahren stattfinden, um das Langfristziel bis 2030 $\mathrm{zu}$ erreichen. Diese Feststellung wird durch das BundesKlimaschutzgesetz unterstützt, das als Rahmengesetz für Klimaschutzmaßnahmen dient ${ }^{92}$ und genauso wie das BEHG auf den Vorschlägen des Klimakabinetts ${ }^{93}$ beruht, weshalb es bei der Auslegung zu berücksichtigen ist: Es bestimmt in $\$ 4$ Abs. $3 \mathrm{~S}$. 1 ausdrücklich, dass eine Abhängigkeit der Jahresemissionsmengen ab dem Jahr 2021 besteht. Über- oder unterschreiten die Treibhausgasemissionen in einem Sektor die entsprechenden Jahresemissionsmengen, so wird die Differenz auf die nächsten Jahre gleichmäßig bis 2030 bzw. bis zum nächsten Zieljahr des folgenden Klimaschutzplans angerechnet. Hieraus ergibt sich eine mengenmäßige Begrenzung, die zwar nicht zwingend im einzelnen Jahr, aber für die Gesamtwirkzeit des Instruments gilt. ${ }^{94}$ Somit liegt eine mengenmäßige Begrenzung vor, die die Abgabe nach dem Gedanken der Vorteilsabschöpfung rechtfertigen kann.

\subsubsection{Rechtfertigung über den Lenkungszweck}

Selbst wenn eine isolierte Teilbetrachtung der Einführungs- und Übergangsphase angezeigt sein sollte und eine mengenmäßige Begrenzung nicht vorläge, könnte eine nichtsteuerliche Abgabe trotzdem durch die angestrebte Lenkungswirkung gerechtfertigt sein. Dass nämlich das Erfordernis einer mengenmäßigen Begrenzung die einzige Möglichkeit ist, zu einer Rechtfertigung der Bewirtschaftung des Nutzungsraums Luft zu gelangen, lässt sich dem Nichtannahmebeschluss zum ZuG 2012 nicht entnehmen. In dem verwaltungsgerichtlichen Verfahren, das dem Nichtannahmebeschluss voranging, hat auch das BVerwG klargestellt, dass ,[e]ine Nutzungsregelung ... aber unterschiedlich ausgestaltet werden [kann]. Neben der hoheitlichen Verleihung begrenzter Nutzungsrechte ist es ebenso denkbar, die Nutzungsmöglichkeit nicht individuell zu limitieren, sondern an eine Entgeltzahlung zu koppeln." 95 Genauso hat das BVerfG in seinem Beschluss zum Wasserpfennig die Möglichkeit der Rechtfertigung einer nichtsteuerlichen Abgabe durch ihre Lenkungswirkung angesprochen, dies aber im Ergebnis offen gelassen, da es in der Erhebung von Wasserentnahmeentgelten Vorteilsabschöpfungsabgaben sah. ${ }^{96} \mathrm{Im}$ Nichtannahmebeschluss zu Regelungen des ZuG 2012 stützt das BVerfG die Rechtfertigung über eine mengenmäßige Begrenzung explizit auf die Notwendigkeit einer Preisbildung, die zu der gewünschten Lenkungswirkung führen soll. Mit anderen Worten: Eine Rechtfertigung einer nichtsteuerlichen Abgabe kann bereits dann in Betracht kommen, wenn durch eine Preisbildung gesichert ist, dass sie Lenkungswirkung entfaltet. Vor diesem Hintergrund erklärt sich das Erfordernis der mengenmäßigen Begrenzung im Bereich des EU-ETS da- mit, dass auch in der zweiten Handelsperiode des EU-ETS noch rund 90 Prozent der Zertifikate kostenlos zugeteilt wurden. Bei einer fehlenden mengenmäßigen Begrenzung wäre somit für den Großteil der in Deutschland nach $\$ 16$ S. 1 ZuG 2012 kostenlos zugeteilten Zertifikate gar kein Preissignal für Emissionen entstanden. Nur für 40 Millionen Berechtigungen, die nach $\$ 19$ S. 1 ZuG 2012 veräuBert wurden, hätte ein ursprüngliches Preissignal eine Lenkungswirkung entfalten können. Da es bei einer fehlenden mengenmäßigen Begrenzung aber gar nicht zum Zukauf solcher Zertifikate gekommen wäre, hätte es vollkommen an einer preislichen Lenkungswirkung gefehlt. Eine Rechtfertigung einer nichtsteuerlichen Abgabe über die Lenkungswirkung eines Preises trotz fehlender mengenmäßiger Begrenzung kam im Bereich des EU-ETS nach damaliger Ausgestaltung deshalb nicht in Betracht.

Anders verhält es sich aber im System des BEHG, bei dem die Zertifikate von Beginn an ersteigert werden müssen. Hiermit ist automatisch ein Preissignal gegeben, das zu einer entsprechenden Lenkungswirkung führt. ${ }^{97}$ Einem solchen Preissignal - mag es auch aus ökonomischer Sicht nicht optimal sein ${ }^{98}$ - kann gerade unter Berücksichtigung der weitaus geringeren Zertifikatspreise in der Anfangszeit des EU-ETS ${ }^{99}$ aus rechtlicher Perspektive sicherlich nicht entgegengehalten werden, dass die Legislative ihre Einschätzungsprärogative ${ }^{100}$ in Bezug auf die Lenkungswirkung überschritten habe.

\section{Zusammenfassung und Würdigung}

Bei der durch das BEHG konstituierten Abgabe handelt es sich auch in der Einführungs- und Übergangsphase bis 2027 trotz des Verkaufs der Zertifikate zu einem Fixpreis bzw. einer Preisobergrenze um keine Steuer, weil der Erhalt der Zertifikate eine Gegenleistung darstellt. Die Abgabe kann als Sonderabgabe im weiteren Sinn eingeordnet werden und ist insofern von der Sachkompetenz des Art. 74 Abs. 1 Nr. 24 GG gedeckt. Argumentationslinien,

91) BT-Drs. 19/14746, S. $32 \mathrm{ff}$

92) So ausdrücklich BT-Drs. 19/14337, S. 17.

93) Siehe: Eckpunkte für das Klimaschutzprogramm 2030, Fassung nach Klimakabinett, Stand 17.7.2020, abrufbar unter https:// www.bmu.de/fileadmin/Daten_BMU/Download_PDF/Klimaschutz/eckpunkte_klimaschutzprogramm_2030.pdf, S. $20 \mathrm{f}$.

94) Dass die im KSG enthaltenen Mechanismen auf das BEHG Anwendung finden, vertritt auch das Bundesministerium für Umwelt: PlPr. 19/169, S. 21111.

95) BVerwG, Urt. v. 10.10.2012 - 7 C 8.10, BeckRS 2013, 46298, Rdnr. 30.

96) BVerfG, Beschl. v. 7.11.1995 - 2 BvR 413/88, 1300/93, BVerfGE 93, 319, 345 f., das Vorliegen einer Vorteilsabschöpfungsabgabe trotz Fehlens einer mengenmäßigen Beschränkung der Wasserentnahme kann wohl mit dem dortigen Erlaubnisvorbehalt erklärt werden, vgl. Klinski/Keimeyer, ZUR 2020, 342, 347.

97) Siehe dazu auch: Frenz, DVB1. 2007, 1385 mit Verweis auf BVerfG, Beschl. v. 19.7.2000 - 1 BvR 539/96, BVerfGE 102, 197, $217 \mathrm{f}$.

98) Der $\mathrm{CO}_{2}$-Preis sollte zum Erreichen des 2-Grad-Ziels selbst bei nicht vollständiger Einpreisung der sozialen Kosten von Kohlenstoffemissionen im mittleren zweistelligen Bereich liegen, vgl. Edenhofer/Flachsland/Kalkuhl/Knopf/Pahle, Optionen für eine $\mathrm{CO}_{2}$-Preisreform, MCC-PIK-Expertise für den Sachverständigenrat zur Begutachtung der gesamtwirtschaftlichen Situation, 2019, S. 22 f., m.w. N.; so auch: Matthes, Der Preis auf $\mathrm{CO}_{2}$, Heinrich Böll Stiftung - Schriften zur Ökologie, Bd. 48, S. $30 \mathrm{ff}$.

99) Siehe hierzu: Umweltbundesamt/Deutsche Emissionshandelsstelle, Emissionshandel: Auswertung der ersten Handelsperiode 2005-2007, 2009, S. $94 \mathrm{ff}$.

100) Zum Begriff der Einschätzungsprärogative: BVerfG, Beschl. v. 29.10.1987 - 2 BvR 624, 1080, 2029/83, BVerfGE 77, 170, $214 \mathrm{f}$. 
die eine Rechtfertigung einer solchen Abgabe erlauben, sind ebenfalls vorhanden, sodass dem BEHG die Verfassungswidrigkeit nicht derart offensichtlich auf die Stirn geschrieben steht, wie es teilweise suggeriert wird. Erfreulich wäre es deshalb, wenn das Instrument aus der juristischen Perspektive offener aufgenommen würde und sich die Diskussion zu einzelnen Ausgestaltungsmerkmalen verschöbe, die noch rechtliche und ökonomische Fragen aufwerfen. ${ }^{101}$

Open Access. Dieser Artikel wird unter der Creative Commons Namensnennung 4.0 International Lizenz veröffentlicht, welche die Nutzung, Vervielfältigung, Bearbeitung, Verbreitung und Wiedergabe in jeglichem Medium und Format erlaubt, sofern Sie den/die ursprünglichen Autor(en) und die Quelle ordnungsgemäß nennen, einen Link zur Creative Commons Lizenz beifügen und angeben, ob Änderungen vorgenommen wurden.

Die in diesem Artikel enthaltenen Bilder und sonstiges Drittmaterial unterliegen ebenfalls der genannten Creative Commons Lizenz, so- fern sich aus der Abbildungslegende nichts anderes ergibt. Sofern das betreffende Material nicht unter der genannten Creative Commons Lizenz steht und die betreffende Handlung nicht nach gesetzlichen Vorschriften erlaubt ist, ist für die oben aufgeführten Weiterverwendungen des Materials die Einwilligung des jeweiligen Rechteinhabers einzuholen.

Weitere Details zur Lizenz entnehmen Sie bitte der Lizenzinformation auf http://creativecommons.org/licenses/by/4.0/deed.de.

Open Access funding enabled and organized by Projekt DEAL.

101) Angesprochen sind hiermit neben der Preisdiskussion z.B Fragen der Sozialverträglichkeit sowie der Zusammenführung mit dem EU-ETS, vgl. Edenhofer/Flachsland/Kalkuhl/Knopf/ Pahle, Bewertung des Klimapakets und nächste Schritte: $\mathrm{CO}_{2}^{-}$ Preis, sozialer Ausgleich, Europa, Monitoring, MCC-PIK, 2019.

\section{Negativemissionstechnologien als neues Instrument der Klimapolitik: Charakterisiken und klimapolitische Hintergründe ${ }^{1}$}

Till Markus, Romina Schaller, Erik Gawel, Klaas Korte

(C) Der/die Autor(en) 2021. Dieser Artikel ist eine Open-Access-Publikation.

Der ${ }^{1}$ Weltklimarat IPCC zeigt in seinem Sonderbericht „1,5 ${ }^{\circ} \mathrm{C}$ globale Erwärmung“ aus dem Jahr 2018, dass das Erreichen der im Pariser Übereinkommen vereinbarten Klimaziele nach wie vor möglich ist. Dies erfordere allerdings nicht nur einen grundlegenden Wandel unserer Lebens- und Wirtschaftsweise sowie die Reduktion von Treibhausgasemissionen auf Null (spätestens in der zweiten Hälfte dieses Jahrhunderts), sondern auch die dauerhafte Entnahme erheblicher Mengen von $\mathrm{CO}_{2}$ aus der Atmosphäre. Letzteres kann durch eine Reihe unterschiedlicher Verfahren und Technologien erreicht werden, sogenannte Negativemissionstechnologien (NETs). Der folgende zweigeteilte - Beitrag untersucht zentrale klimawissenschaftliche, -politische und -rechtliche Aspekte eines möglichen Einsatzes von Negativemissionstechnologien als Instrument der Klimapolitik. Hierzu werden aktuell diskutierte NETs vorgestellt und wesentliche Unterschiede $\mathrm{zu}$ anderen klimapolitischen Maßnahmen herausgearbeitet. Es zeigt sich, dass eine undifferenzierte Subsumtion von NETs unter den Begriff der Abschwächung (Mitigation) zu einer Verlagerung der Klimaschutzbemühungen führen kann, d.h. weg von Maßnahmen, die eine Vermeidung der Entstehung von Treibhausgasen bewirken, hin zu Verfahren

PD Dr. Till Markus, LL.M.,

Dr. Romina Schaller, LL.M. Eur.,

Department Umwelt- und Planungsrecht,

Helmholtz-Zentrum für Umweltforschung,

Leipzig, Deutschland

Prof. Dr. Erik Gawel,

Klaas Korte, Dipl.-Ök.,

Department Ökonomie,

Helmholtz-Zentrum für Umweltforschung,

Leipzig, Deutschland und Technologien, deren Beitrag zur Verwirklichung der Klimaschutzziele derzeit noch unsicher ist und die z. T. mit erheblichen Risiken behaftet sind.

\section{Einleitung und Hintergründe}

Die Vertragsparteien der Klimarahmenkonvention (KRK) einigten sich bereits im Jahr 1992 auf die Begrenzung der atmosphärischen Treibhausgaskonzentrationen. Zweck dieser Vereinbarung war und ist die Vermeidung einer ,gefährlichen anthropogenen Störung des Klimasystems".2 ${ }^{2}$ Ende 2015 wurde im Rahmen des Pariser Übereinkommens (PÜ) weitergehend vereinbart, dass der durch den Treibhauseffekt verursachte Anstieg der Erdtemperatur ,deutlich unter $2^{\circ} \mathrm{C}$ über dem vorindustriellen Niveau gehalten wird". 3 Weitere Anstrengungen sollen unternommen werden, um den Temperaturanstieg auf $1,5^{\circ} \mathrm{C}$ zu begrenzen. ${ }^{4}$ Zur Einhaltung der Erwärmungsobergrenze legt das PÜ darüber hinaus fest, dass der Scheitelpunkt der Treibhausgasemissionen ,so bald wie möglich“ erreicht werden

1) Hinweis: Der Beitrag ist im Rahmen der Helmholtz-Klima-Initiative (HI-CAM) erarbeitet worden. HI-CAM wurde mit Mitteln des Impuls- und Vernetzungsfonds der Helmholtz-Gemeinschaft (IVF) gefördert. Die Verantwortung für den Inhalt dieser Veröffentlichung liegt bei der Autorin und den Autoren.

2) Art. 2 S. 1 Rahmenübereinkommen der Vereinten Nationen über Klimaänderungen (Klimarahmenkonvention - KRK), BGBl. II, S. 1784 .

3) Art. 2 Abs. 1 lit. a PÜ. Zur Entwicklung der $2^{\circ}$ Celsius-Leitplanke, siehe Schlacke, Grenzwert oder Politikziel? Dogmatik und Legitimität der $2^{\circ}$ Celsius-Leitplanke, in Dilling/Markus, Ex Rerum Natura Ius? Recht aus der Natur der Sache, 2014, S. 93.

4) $1,5^{\circ} \mathrm{C}$, über dem vorindustriellen Niveau“, siehe Art. 2 Abs. 1 lit. a PÜ. 